\title{
The Effect of Tai Chi on the Low Back Pain and Disability Among Older Adults
}

\author{
$1^{\text {st }}$ Lalaine Melissa P. Jimenez \\ Saint Louis University School of Nursing \\ Bonifacio St., Baguio City \\ Baguio City, The Philippines \\ lalainejimenez@ymail.com \\ 4th Nicole C. Banayat \\ Saint Louis University School of Nursing \\ Bonifacio St., Baguio City \\ Baguio City, The Philippines \\ nicolebanayat@ymail.com \\ 7th Jenica S. Mandagan \\ Saint Louis University School of Nursing \\ Bonifacio St., Baguio City \\ mandagan.jenica@yahoo.com \\ 10th Ivy Ruth E. Tami-ing \\ Saint Louis University School of Nursing \\ Bonifacio St., Baguio City \\ ivytami@yahoo.com
}

\author{
2nd Eric C. Jacinto \\ Saint Louis University School of Nursing \\ Bonifacio St., Baguio City Baguio City, \\ The Philippines \\ ericj_0811@yahoo.com \\ 5th Judy Ann N. Canaveral \\ Saint Louis University School of Nursing \\ Bonifacio St., Baguio City \\ Baguio City, The Philippines \\ judyanncanaveral_05@yahoo.com
}

8th Alisa Ruth B. Ponceja

Saint Louis University School of Nursing

Bonifacio St., Baguio City

alisa.ponceja@ymail.com

\author{
3rd Jason Sherwin Angelo Y. Ramirez \\ Saint Louis University School of Nursing \\ Bonifacio St., Baguio City \\ Baguio City, The Philippines \\ jsayramirez@gmail.com \\ 6th Jovelyn P. Gruspe \\ Saint Louis University School of Nursing \\ Bonifacio St., Baguio City \\ Baguio City, The Philippines \\ akikosy5683@yahoo.com.ph \\ 9th Kedzee B. Sibayan \\ Saint Louis University School of Nursing \\ Bonifacio St., Baguio City \\ kedzsibayan@gmail.com
}

\begin{abstract}
Low back pain (LBP) is a therapeutically demanding condition among older adults. This quasiexperimental study aimed to determine the effect of tai chi, a non-pharmacologic intervention for pain, on older adults' degree of $\mathrm{LBP}$ and disability. A sample of 90 older adults was non-randomly divided into experimental group $(n=45)$ and control group $(n=45)$. The former received tai chi sessions for 14 days (40 minutes daily), with degree of LBP (Visual Analogue Scale) and disability (The Revised Oswestry Disability Index) examined at baseline and at $2^{\text {nd }}$ week. Both groups also accomplished daily activity logs. T-test analysis revealed a significant decrease in the degree of LBP in both groups but experimental group's improvement was greater $(p<0.05)$. Additionally, only the experimental group demonstrated a significant reduction in disability index scores $(p<0.05)$. In conclusion, tai chi is an effective complementary management of LBP that promotes independence in the performance of ADLs among older adults.
\end{abstract}

Keywords: tai chi, VAS, revised Oswestry disability index, older adults, low back pain

\section{INTRODUCTION}

The aging process is a course of a biological reality largely beyond human control. One of the most common and potentially disabling conditions among older adults is low back pain (Rudy, Weiner, Lieber, Slaboda, \& Boston, 2007). It has often been considered as a constellation of symptoms, with mechanical LBP being the most prevalent because of its complexity (Jarvik \& Deo, 2002). In addition to physiological age-related changes, its development has been suggested to be associated with postural load conditions reflecting the lifestyle of individuals (Yamamoto, 2003).

Part of assessing the problem is considering pain duration, which is a critical defining feature of chronic low back pain (CLBP). Individuals with CLBP has a low back pain persisting for longer than 7-12 weeks which is most commonly characterized by recurrences and repeated episodes (Pfleger, 2003) . In 2006, 5.9 
million (13.6\%) aging 18-85 years in the Philippines suffered from chronic pain. $21 \%$ of those who are above 50 years old suffer from pain in the lower back. Gender differences in pain location also revealed that $26 \%$ of men and $23 \%$ of women reported to suffer more pain in the [1]. In addition, about thirty-six percent of communitydwelling older adults experience an episode of back pain per year and of those reporting an episode, $21 \%$ reported moderate to severe pain that occurs very often or more [2]. As the population ages, the disease burden of low back pain will also increase substantially among senior citizens. Reflecting the increasing size of the older population, National Statistics Office, Census of Population and Housing $(\mathrm{CPH})$ reported that the population of older people (60 and above) residing in Baguio grew to 18, 060 as of 2010 [3],[4].

While many older adults remain functional despite low back pain [5], sufferers may still experience restrictions in their activities of daily living and a reduction in their independence in fulfilling their own needs (Walker, Sofaer, \& Holloway, 2006) . Basic activities of daily living (ADLs) are very important for the older adults because majority of them still enjoys independence till the age of 79 (McDowell and Newell, 1996).

Consequences of low back pain among communitydwelling adults highlight difficulty in doing activities of daily living, such as walking, lifting objects and taking a bath, greater difficulties in social interaction are well documented in literatures [6]. It was also found to be associated with difficulty in standing in one place, pushing or pulling a large object, and walking a half-mile [7]. Similarly, investigation reveals that Visual Analogue Scale scores of an older adults group, when sitting, was lower, indicating that a characteristic of nonspecific low back pain in older adults patients may be less pain in a sitting posture [8]. This supports the clinical impression that older adult patients may have greater intensity of low back pain while in motion or standing, but less pain when sitting. Furthermore, low back pain was found to affect numerous areas of psychosocial functioning in older individuals including relationships, self-identity, emotions, social and family roles and life contentment (Snelgrove \& Liossi, 2009) . Besides suffering from strains in relationship, low back pain also correlates positively with a disturbed sleep pattern [9], [10], [11], generally lowering sleep efficiency, decreasing total sleep time, and increasing duration of sleep onset [12]. Higher levels of anger and frustration may be due to this lack of sleep (Hui Lin, et al., 2014). Moreover, intermittent increases in pain can also markedly alter disability. While pain intensity can affect disability, the recurrent nature of low back pain also affects the ability of an individual's function in both work and personal life [13]. Such functional impairments place the older adults in a vulnerable state, diminishing their ability to perform personal-care tasks (Manoochehri et al., 2014). Conclusively, low back pain is a physical distress in the population of the older adults leading to a decline in health overtime. This negative experience poses an adverse impact in older individuals' well-being and quality of life.

Tai chi is a predominant form of exercise in urban China, particularly among those with older age and chronic medical diseases [14]. Evidence suggests benefits including: improved strength, conditioning, coordination, and flexibility. Tai chi can also enhance the overall flexibility and psychological well-being since it is known that stress reduction often occurs when individuals indulge in activities [15]. Being branded as a safe method of exercise in older adults and patients with neurological disorders [16], tai chi is shown to reduce pain and stiffness, enhance sleep, give better balance, and increase awareness, calmness, and an overall sense of wellbeing [17]. Supporting research evidences on tai chi's therapeutic effects suggest that it can be justifiably implemented in the clinical setting on a wide-scale basis [18]. This implies that tai chi has the potential to minimize functional disability.

The theoretical framework of the study is Orems' Self-Care Deficit General Theory of Nursing. Attending to the effects of pathologic and age-associated low back pain and maintaining functional status can be viewed as therapeutic self-care demands of the older adults. In addition, their self-care agency becomes impaired as their ability to provide continuous effective self-care become limited due to low back pain. If there is a self-care deficit between what the individual can do and what needs to be done to maintain optimum functioning, then nursing is needed [19]. Thus, tai chi, as a self-care measure, will be incorporated into the older adults' self-care system, "the performance of which, requires specialized knowledge and skills to be acquired through training and experience" [20].

The intervention's two-fold phase represents the pained and disabled participants' transition from a partly compensatory system to a supportive-educative system as the tai chi sessions advance from supervised to unsupervised. Receiving supervised tai chi session is a situation represented by the partly compensatory system, in which "both the nurse and patient may have the major role in the performance of self-care measures" [20]. Conversely, the unsupervised tai chi sessions exemplifies a supportive educative system [20]. It reinforces the 
nurses' role to monitor and assist the older adults to determine if self-care measures (tai chi) were effectively performed. Overall, the nurse's role is to promote the patient as a self-care agent and overcome human limitations [19].

In order to acknowledge the self-care requisites and the extent to which the self-care agency is affected, the Revised Oswestry Disability Index features the influences of pain to the older adults' ability to manage everyday activities. An improvement of research's primary outcome measures will indicate a successful attainment of client structural integrity and human functioning, which would imply that tai chi is a therapeutic nursing intervention in meeting self-care needs and in designing a system that would best meet the needs and augment the self-care abilities of older adults.

The study aimed to determine the effects of tai chi on the low back pain and disability index score of the older adults in the experimental group prior to introduction of tai chi and the significant difference in the degree of low back pain and disability index between the experimental and control group.

The findings will make available new evidence to support advantageous effect of tai chi in addressing disabilities, enhancing the evidence-based knowledge on complementary and alternative interventions for low back pain. This study may also help barangays in the implementation of Republic Act No. 6847 through integration of tai chi and a wellness- promoting information guide in their barangay fitness program.

\section{METHODS}

This study utilized a quasi-experimental, pre-test and post-test design, with non-randomization of 90 participants to an experimental and a control group. This study was conducted in the selected areas of Baguio City, the Philippines. A non-random referral and convenience sampling was utilized to attain the desired sample population of the experimental and control group.

Inclusion criteria were: age 60 and above, with localized intermittent low back pain for the last 6 months, and at pretest, no walking aids, independent in their activities of daily living, are able to communicate and have no previous experience in tai chi. Exclusion criteria were: severe cognitive impairments, symptomatic cardiovascular disease at moderate exertion level, poorly controlled hypertension, orthostatic hypotension, and diagnosis of stroke, peripheral neuropathy, osteoporosis, crippling arthritis, metastatic cancer, those with current participation in other instructor-led and or self-paced exercise programs, and disability index score of $61 \%$ and above.
Of the 101 participants $(n=101)$ eligible for the study, 56 decided to be included to the experimental group and 45 were included to the control group. Of the 56 participants in the experimental group, 11 participants were excluded due to 2 consecutive absences or 3 nonconsecutive absences during the course of the study, generating an attrition rate of $19.64 \%$. Follow-up rate at 2 weeks after baseline was $100 \%$ for the control group.

This study utilized two data gathering tools, namely: Visual Analogue Scale and The Revised Oswestry Disability Index for low back pain/dysfunction. A reduction in the degree of low back pain is of clinically meaningful significance [21]. Farrar et al [22] further elucidates that $30 \%$ reduction in the degree of low back pain indicates a "much improved" clinically meaningful change while a $50 \%$ reduction indicates a "very much improved" clinically meaningfully changed.

The Revised Oswestry Disability Index for low back pain/ dysfunction has been considered as a gold standard in determining functional disability outcome measures for low back pain. Fairbank et al [23] proposes that Minimum Detectable Change (at 90\% confidence) is 10 points. That means at least a $10 \%$ change is required to be clinically meaningful. The Revised Oswestry Disability Index (for low back pain/ dysfunction) is composed of section 1section 10; each section has 6 possible answers. Statement 1 is graded as 0 points: statement 6 is graded as 5 points. Disability scores will be interpreted as: 0\%-20\%: Minimal disability, 21\%-40\%: Moderate disability, 41\%60\%: Severe disability, 61\%-80\%: Crippled, and 81$100 \%$. The researchers secured both Tagalog and Ilocano translations of the Revised Oswestry Disability Index Questionnaires.

Daily activity $\operatorname{logs}$ were also given where they recorded their activities of the day, medications, and other therapies to address low back pain. Participants were asked to record their socialization activities in the morning, lunch, and afternoon, including their hours of sleep and naps in a day.

\section{A. General procedures Were as Follows}

1. The researchers underwent Yang style 10 forms tai chi training course for four Sundays from certified tai chi trainers of the Wushu Federation of the Philippines-Baguio Chapter.

2. Communication letters were sent to Barangay Captains of the selected communities, inclusive of inquiries to their respective Barangay Senior Citizens Association (BSCA) for older adult living within their vicinity.

3. With granted permission, a letter of invitation to participate in the research was given. The researchers 
also determined the older adults' eligibility to participate and made certain that they identify the location of back pain.

4. A copy of the informed consent was given and discussed to the prospective participants who were able to meet the inclusion criteria. Written consent was obtained. Confidentiality and anonymity of the collected information were also guaranteed. If the participants have any questions, concerns or complaints about the study, they were encouraged to reach the researchers through the contact information provided in the informed consent. After the discussion of the written informed consent, a tai chi video was shown to them. Discussion of the Visual Analogue Scale, the Revised Oswestry Disability Index and the Daily Activity log was integrated in the discussion of Part I: Information Sheet of the written informed consent form.

5. They were also instructed to refrain from doing activities that may worsen their low back pain such as lifting heavy objects or sudden twisting of the trunk during the 2 weeks implementation period.

6. The participants were given time to decide whether or not to participate in the study. Once the participants decided to take part in the study, they were asked to select their group of choice, whether in the experimental or control group. Thereafter, they were asked to accomplish Part II of the written informed consent.

B. Research Protocol for the Experimental Group:

1. The researchers reintroduced. Donning proper attire, importance of warming up, stretching and cooling down were discussed.

2. On the first day of the Tai Chi session, the degree of pain and disability index score were measured using the Visual Analogue Scale and The Revised Oswestry Disability Index 10 minutes before the actual implementation. Afterwards, the participants were informed of their scores. The scores were recorded on a VAS Score and Disability Level Record Form. Each participant was also given a daily activity $\log$.

3. Tai chi exercises were performed over a 2 week period (7 times per week, 40 minutes of tai chi exercise per session) in the morning or in the afternoon. The researchers supervised the performance of tai chi on the first six (6) days of implementation.

4. The researchers sent a daily SMS reminder of the tai chi exercise and filling up of daily activity log. In the event that a session was missed, the participant was allowed to compensate by having another session at the end of the second week of tai chi.

5. On the $14^{\text {th }}$ day, low back pain and disability index score were reevaluated after the performance of tai chi.

\section{Research Protocol for the Control Group}

1. On baseline visit, the researchers reintroduced selves and reminded the content of the written consent form.

2. The degree of pain and disability index was measured using the Visual Analogue Scale and The Revised Oswestry Disability Index on the $1^{\text {st }}$ day; they were informed of their pre-test scores. The scores were recorded on a Visual Analogue Scale Score and Disability Level Record Form. Each participant was also given a daily activity log.

3. The participants were instructed to perform their usual activities of daily living but to refrain from doing activities that may increase low back pain. The older adults were not taught of the steps and were not required to perform tai chi.

4. The participants were reminded daily via SMS to fill up the daily activity $\log$.

5. On the $14^{\text {th }}$ day of the research, the degree of low back pain and disability index score were reevaluated using the VAS and the Revised Oswestry Disability Index for low back pain/ dysfunction.

The data were analyzed by comparing the treatments with respect to their pre-test and post-test measurements. Microsoft Excel 2010 was used to calculate the differences between the two dependent group means in the pre and post evaluations, using related t-test. To identify any difference in effect between the experimental and control group, unrelated t-test was also used.

\section{RESULTS AND DISCUSSION}

In order to determine the effects of tai chi on the degree of low back pain and disability index score of the older adults, pretest and posttest scores of outcome measures degree of low back pain (Visual Analogue Scale) and disability (The Revised Oswestry Disability Index) examined at baseline and at $2^{\text {nd }}$ week of the study were compared.

Table 1 presents the effects of tai chi on the degree of low back pain and disability index in the experimental group. The pretest mean VAS score of the group is 31.06 and the posttest mean degree of low back pain score is 6.37 , with a mean difference of 24.68 which demonstrates a reduction in the degree of low back pain. The pretest mean disability index score of the group is 16.48 and the posttest mean disability index score is 9.6 , with a mean difference of 6.88 , which also shows a reduction in 
disability index. After employing paired t-stat analysis in degree of the low back pain, with a DF of 44, the t-stat is 5.63 and the two tailed p-value is less than 0.0001. By conventional criteria, this difference is considered to be statistically significant. Similar analysis for disability index, with a DF of 44 , resulted to a t-test of 4.17 , with a two-tailed $\mathrm{P}$ value equal to 0.0001 revealing extremely statistically significant by conventional criteria.

TABLE 1. EFFECT OF TAI CHI TO OLDER ADULTS' LOW BACK PAIN AND DISABILITY INDEX IN THE EXPERIMENTAL

\begin{tabular}{|c|c|c|c|c|c|}
\hline & Pretest & Posttest & $\begin{array}{c}\text { Mean } \\
\text { Difference }\end{array}$ & $\begin{array}{l}\text { T- } \\
\text { test }\end{array}$ & P-value \\
\hline $\begin{array}{l}\text { Degree of low } \\
\text { back pain }\end{array}$ & 31.07 & 6.38 & 24.68 & 5.63 & $\begin{array}{c}< \\
0.0001^{*}\end{array}$ \\
\hline Disability Index & 16.48 & 9.6 & 6.88 & 4.17 & $0.0001 *$ \\
\hline significant $\alpha=0.0$ & \multicolumn{4}{|c|}{$*$ p-values $<0.05$ are statistically } & \\
\hline
\end{tabular}

Pretest scores indicating existence of low back pain can be attributed to the decline of pain modulation mechanism. Studies found that the efficacy of endogenous pain control mechanisms actually decreases in older adults, possibly contributing to the high prevalence of their pain [24], [25].

A radiography study reported an increased peripheral vasodilation and hand temperature during tai chi exercise [26], suggesting a relaxation response mechanism. This finding is consistent with results of a study attributing the proportion of the observed $30 \%$ reduction in pain intensity to the activation of relaxation responses in the performance of tai chi among 160 volunteers between ages 18 and 70 years with persistent nonspecific low back pain [27]. These relaxation responses follow a decreased tension on the paraspinal muscles, which then lessen the compressive forces on the spine [28]. Therefore, the decreased likelihood of muscular tension can be associated with the beneficial decrease in the degree of low back pain.

In this study, comparison of average mean per section of the disability index score before and after introduction of tai chi showed a consistent reduction of disability index score in all areas: pain intensity, personal care, and lifting, walking, sitting, standing, sleeping, social life, travelling, and changing degree of pain. Only few studies have concentrated on defining the impacts of tai chi on functions, especially some identified in the Revised Oswestry Disability Index, such as personal care, travelling and social life among others. Yet, improving physical deficits, particularly in balance capacity (Hong et al., 2000; Tse \& Bailey, 1992; Wong et al., 1996) could have a direct causal pathway in improving functional mobility, such as standing, sitting, and walking. It has also been suggested that the practice of tai chi may enhance the repertoire of motor programs stored in the brain (Tse \& Bailer, 1992) and therefore serve to train various balance systems to promote greater steadiness. Moreover, the constant weight shifting and body rotations observed during tai chi increase load on the lower limbs (Philip et al., 2012) thereby improving or maintaining the eccentric strength of leg muscles needed for standing balance [29] or in general, activities involving the lower extremities. Tai chi also improves muscle coordination [30], promotes better circulation and increases range of motion in joints and muscles [31], functional movements that are presumably needed in accomplishing everyday tasks. Sleep quality and duration was also determined to have improved in people who had been using tai chi [32], [33]

Furthermore, limiting physical activity is known to be a natural strategy for individuals with certain acute pain conditions (Jensen, Moore, Bockow, Ehde, \& Engel, 2011). This avoidance is a maladaptive response where patients avoid certain activities that they anticipate to cause an increase in pain and suffering [34]. Despite being part of their pain management, its effects on pain perception renders it inappropriate. This extreme response of "avoidance" strategy is thought to produce a number of psychological and physical consequences which promote the development of the invalid status and exaggerated pain perception (Lethem, Slade, Troup \& Bentley, 1983). Therefore, if functional disability occurs at least in part because of low back pain, then it is plausible to believe that exercises that decrease pain might result in improved disability status.

To determine the significant differences in the degree of low back pain and disability index in-between groups, their respective difference scores between pretest and posttest measurements were compared.

Table 2 presents the effects of tai chi on the degree of low back pain and disability index in-between groups. The mean degree of low back pain score difference in the experimental and control are 24.69 and 8.87, respectively. Mean difference of disability index scores for experimental and control are 6.89 and 0.89 respectively. Employing the t-test analysis of mean differences on the degree of low back pain, with a DF of 44, shows a t-stat of 6.264 and a two-tailed p value of less than 0.0001. By conventional criteria, this is considered to be statistically significant, suggesting a significant difference in the mean disability index scores in-between groups. The t-test analysis of mean differences of disability index scores, with a DF of 44, shows a t-stat of 2.78 and a two-tailed pvalue equal to 0.0080 . By conventional criteria, this 
difference is also considered to be very statistically significant, suggesting a significant difference in the disability index score in-between groups.

TABLE 2. EFFECT OF TAI CHI TO OLDER ADULTS' LOW BACK PAIN AND DISABILITY INDEX IN THE EXPERIMENTAL GROUP AND CONTROL GROUP.

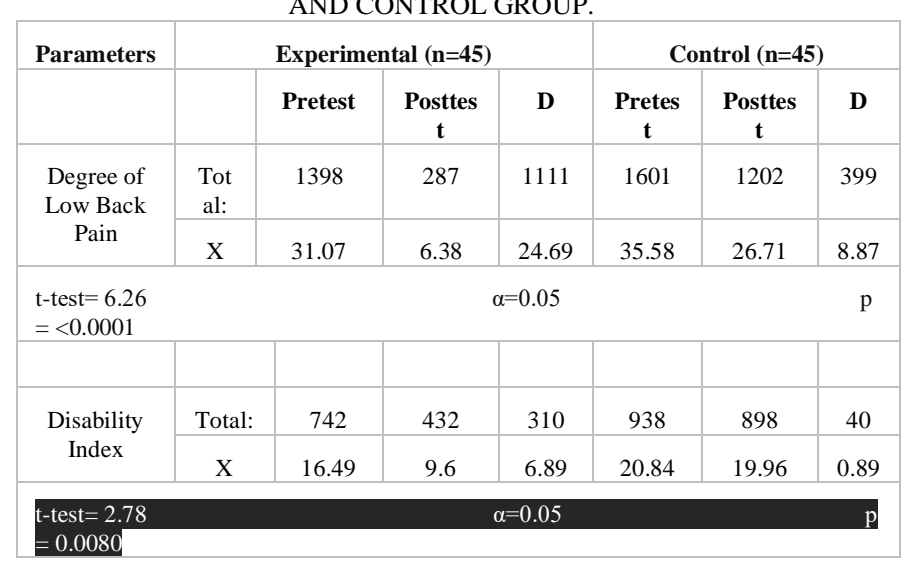

In this study, the experimental group demonstrated a $79.46 \%$ reduction in the degree of their low back pain, which, according to Farrar et al [22], can be equated to a "very much improved" clinically meaningfully change with respect to its value being above the proposed 50\% cut-off. Additionally, previous literature suggests that a $30 \%$ reduction in pain related outcomes indicates an estimate of a clinically worthwhile effect [35]. Conversely, progress of control group's degree of low back pain from pretest to posttest revealed a $33.17 \%$ reduction. This further contradicted our expectations because the control group exhibited a significant decrease in degree of low back pain like the experimental group with a mean difference of 8.87. This is a "much" improved clinically meaningful change [22], but it would still be reasonable to conclude that, on the basis of the reduction of degree of low back pain in-between groups, the experimental groups exhibited a greater improvement.

The researchers also infer that the concurrence of reduction of low back pain in both groups can be attributed to the use of alternative means to manage low back pain. Data gathered from the daily activity log of the experimental group revealed occasional use of analgesic and anti-inflammatory medications during the tai chi treatment period, which made tai chi a complementary and not an alternative intervention addressing the set parameters. On the other hand, data gathered from the activity $\operatorname{logs}$ of the control group revealed use of medication and non-pharmacological interventions like massage. The unrestricted use of pharmacologic and nonpharmacologic interventions to manage back pain could have contributed to the significantly decreased degree of low back pain of the control group which, the researchers were not able to control for the reason that premium was given to the principle of beneficence. Even so, t-test of mean differences shows that there is a significant difference in the changes in the degree of low back pain and disability index for experimental and control groups.

While there was a reduction in the degree of low back pain, the control group, having a mean difference of 0.89 , had not shown a significant change in their disability index score. Disability index scores of the experimental, on the other hand, faired significant in contrast with the control group. A qualitative study attempted to describe the subjective experiences of people with low back pain and found that their emotional distress and difficulty of doing activities emanated from the pain (Vroman, Warner \& Chamberlain, 2009). Interestingly, Xu et al. (1997) found that participants who performed tai chi articulated "increased awareness of the body" and "of different facets of well-being", reduced stress, and feeling "as though [they] had more energy and strength"'. Activities of daily life for tai chi subjects were also reportedly influenced by the improved "focus," or concentration they had gained, which in turn helped them to slow down.

In this study, participants in the experimental group verbalized pain relief and improved functional abilities after tai chi. The improvement in disability index is also attributed in the perception of tai chi as helpful in alleviating symptoms related to back pain, and thus, amplifying their confidence in managing their low back pain. According to [36], patients who expect back problem to last a long time and hold weak beliefs and confidence in their own ability to control their back problem are often noted to have poorer clinical outcomes.

Furthermore, the experimental group likewise showed improvement in several functional status measures ranging from daily activities such as walking and lifting to moderate-vigorous activities such as running ( $\mathrm{Li}$ et al., 2001). The results show the complementary role of tai chi in the improvement of self-reported physical functioning limitations among physically inactive older individuals. The exclusivity of results to the experimental group may be attributed to tai chi body awareness and relaxation component. It has been suggested that those who perform tai chi has a better and improved cognitive appraisal about their back pain and associated psychological distress (Wayne et al., 2008). When an individual is able to form a representation of the illness and subsequently adopt behaviors to cope with the illness, then significant improvements patient-relevant activities, such as walking and sitting results (Siemonsma, 2013).

Moreover, the reduction of disability in the control group may be due to the fact that the decrease in the 
intensity of their low back pain, despite being significant, was only a "much improved" clinically meaningful change. There is also a determined moderate correlation between physical activity and disability for persons with chronic low back pain, which indicates that persons with chronic low back pain and high levels of disability are also likely to have low levels of physical activity (Lin et al., 2010). Older adults may limit what they do, either because activity exacerbates the pain (Martin, Hadjistavropoulos, \& McCreary, 2005) or because they are afraid of reinjury or falling (Hübscher, Vogt, Schmidt, Fink, \& Banzer, 2010; Vlaeyen \& Crombez, 1998). Evidence suggests that older people living with pain may restrict, reduce or otherwise alter their physical and social activities (Mackichan et al., 2013). This could also have reinforced their maladaptive illness perceptions, which in turn, may lead to maladaptive behavior (i.e., activity limitations) (Siemonsma, 2013). Such avoidance response may lead to a gradual loss of strength, endurance, and motor control, an exacerbation of the fear and avoidance behaviors, adverse physical and psychological consequences (Lethem et al., 1983; Vlaeyen et al., 1995, 1999) thereby further impairing functional independence.

Several limitations of current study should be noted. First, given that results represent short-term effects, it becomes uncertain whether the beneficial outcomes would persist for a longer period of time. Second, the researchers can never ascertain whether the participants received equal doses of the intervention during the unsupervised period. This is because the participants, after receiving the tai chi instructional DVD copies, could have practiced tai chi at home, thereby increasing intervention dosage. Third, the participants varied in terms of their learning style. Despite standardizing how the forms were taught, the researchers needed to adapt to their needs during the supervised tai chi session. Lastly, the researchers also do not discount the possibility that self-evaluation biases may have occurred, where participants bend their answers to better reflect what "they should feel" after performing tai chi and rather than what they actually feel. The researchers were also not able to control the environment, given that the participants received the interventions at different settings. This led to varying preparations of the participants before tai chi (i.e. 2 barangays did non-vigorous short distance jogging before stretching). Still, there was uniformity in delivery of the $40 \mathrm{~min}$ tai chi session.

\section{CONCLUSION}

Tai chi shows positive effects on pain and disability among older adults. In conclusion, tai chi is an effective complementary management of low back pain among older adults and it promotes independence in the performance of activities of daily living. Thus, community health promoting organizations and student nurses should consider including tai chi in their scope of practice and planned programs to address the growing and potentially disabling problem of low back pain. Future studies should also consider other types of musculoskeletal pain prevalent in similar or another age group, use a larger sample size, and include a long-term follow up to investigate the effectiveness of tai chi. H. LU and F. JAVIER, Prevalence and treatment of chronic pain in the. 2011.

[2] A. Weiner, D. K., Haggerty, C. L., Kritchevsky, S. B., Harris, T., Simonsick, E. M., Nevitt, M., \& Newman, "How Does Low Back Pain Impact Physical Function in Independent, WellFunctioning Older Adults? Evidence from the," 2003.

[3] Philippine Statistics Office - National Statistics Office, "Population of 319 Thousand was Recorded in Baguio City (Results from the 2010 Census of Population and Housing)," 2013.

[4] Philippine Statistics Office - National Statistics Office, "A Quarter of a Million Persons in Baguio City (Results from the 2000 Census of Population and Housing, NSO)," 2002.

[5] T. . Rudy, D. . Weiner, S. . Lieber, J. Slaboda, and J. . bOSTON, "The impact of chronic low back pain on older adults: a comparative study of patients and controls," Pain, vol. 131, no. 3, pp. 293-301, 2007.

[6] R. B. Goertz, C. M., Salsbury, S. A., Vining, R. D., Long, C. R., Andresen, A. A., Jones, M. E., ... \& Wallace, "Collaborative Care for Older Adults with low back pain by family medicine physicians and doctors of chiropractic (COCOA): study protocol for a randomized controlled trial," Trials, vol. 14, no. 18, pp. 6215-14, 2013.

[7] D. T. Edmond, S. L., \& Felson, "Function and back symptoms in older adults," J. Am. Geriatr. Soc., vol. 51, no. 12, pp. 1702-1709, 2003. 
[8] S. Aoki, Y., Sugiura, S., Nakagawa, K., Nakajima, A., Takahashi, H., Ohtori, S., ... \& Nishikawa, "Evaluation of nonspecific low back pain using a new detailed visual analogue scale for patients in motion, standing, and sitting: characterizing nonspecific low back pain in elderly patients," Pain Res. Treat., 2012.

[9] C. Jones, L. D., Pandit, H., \& Lavy, "Back pain in the elderly: A review," Maturitas, vol. 78, no. 4, pp. 258-262, 2014.

[10] E. Nalajala, N., Walls, K., \& Hili, "Insomnia in chronic lower back pain: Non-pharmacological physiotherapy interventions," Int. J. Ther. Rehabil., vol. 20, no. 10, pp. 510-516, 2013.

[11] B. Hong, J. H., Kim, H. D., Shin, H. H., \& Huh, "Assessment of depression, anxiety, sleep disturbance, and quality of life in patients with chronic low back pain in Korea," Korean J. Anesthesiol., vol. 66, no. 6, pp. 444-450, 2014.

[12] C. G. Alsaadi, S. M., McAuley, J. H., Hush, J. M., Bartlett, D. J., McKeough, Z. M., Grunstein, R. R., ... \& Maher, "Assessing sleep disturbance in low back pain: the validity of portable instruments," PLoS One, vol. 9, no. 4, 2014.

[13] S. M. McGorry, R. W., BSPT, B. S. W., Snook, S. H., \& Hsiang, "The relation between pain intensity, disability, and the episodic nature of chronic and recurrent low back pain," Spine (Phila. Pa. 1976)., vol. 25, no. 7, pp. 834-841, 2000.

[14] X. . Birdee, G.S., Cai, H., Xiang, Y-B, Yang, G., Li, H., Gao, Y., ... Shu, "T'aiChi As Exercise Among Middle-Aged And Older adults Chinese In Urban China," J Altern Complement Med, vol. 19, no. 6, pp. 550-7, 2013.

[15] T. Sandlund, E. S., \& Norlander, "The effects of Tai Chi Chuan relaxation and exercise on stress responses and well-being: An overview of research," Int. J. Stress Manag., vol. 7, no. 2, pp. 139-149, 2000.

[16] W. R. Audette, J. F., Jin, Y. S., Newcomer, R., Stein, L., Duncan, G., \& Frontera, "Tai Chi versus brisk walking in elderly women," Age Ageing, vol. 35, no. 4, pp. 388-393, 2006.
[17] C. J. Rhoads, "Mechanism of pain relief through Tai Chi and Qigong," J Pain Reli., vol. 2, no. 115, 2013.

[18] L. Klein, P. J., van Hooydonk, K., Kutlesa, M., Yang, H. W., Hu, S., Ming, Y. J., ... \& Lin, "Therapeutic benefits of tai chi/qigong: An overview and critical review," 2010.

[19] J. . George, "Nursing Theories: The Base for Professional Nursing Practice.(5thed.) Pearson Education South Asia Pte Ltd.," 2002.

[20] D. . Orem, Nursing: Concepts of Practice (6thed.). St. Louis: Mosby.

A. M. Kelly, "The minimum clinically significant difference in visual analogue scale pain score does not differ with severity of pain," Emerg. Med. J., vol. 3, no. 205-207, 18AD.

J. . Farrar, "Clinical importance of changes in chronic pain intensity measured on an 11-point numerical pain rating scale," 2011.

Scientific Spine, "Oswestry Disability Index," 2013.

T. J. Edwards, R. R., Fillingim, R. B., \& Ness, "Age-related differences in endogenous pain modulation: a comparison of diffuse noxious inhibitory controls in healthy older and younger adults," Pain, vol. 101, no. 1, pp. 155-165, 2003.

[25] N. Lariviere, M., Goffaux, P., Marchand, S., \& Julien, "Changes in pain perception and descending inhibitory controls start at middle age in healthy adults," Clin. J. Pain, vol. 23, no. 6, pp. 506-510, 2007.

[26] J. Iuliano, B., Grahn, D., Cao, V., Zhao, B., \& Rose, "Physiologic correlates of T'ai Chi Chuan," J. Altern. Complement. Med., vol. 17, no. 1, pp. 77-81, 2011.

J. Hall, A. M., Maher, C. G., Lam, P., Ferreira, M., \& Latimer, "Tai chi exercise for treatment of pain and disability in people with persistent low back pain: a randomized controlled trial," Arthritis Care Res. (Hoboken)., vol. 63, no. 11, pp. 1576-1583, 2011.

[28] B. Gallagher, "Tai chi chuan and qigong: Physical and mental practice for functional mobility," Top. 
Geriatr. Rehabil., vol. 19, no. 3, pp. 172-182, 2003.

[29] L. Wu, G., Zhao, F., Zhou,X., \& Wei, "Improvement of isokinetic knee extensor strength and reduction of postural sway in the older adults from long-term Tai Chi exercise," Arch. Phys. Med. Rehabil., vol. 83, no. 10, pp. 1364-1369, 2002.

[30] W. Ching, L., Ssu-Yuan, C., Jin-Shin, L., \& Alice May-Kuen, "Tai Chi Chuan in Medicine and Health Promotion," Evidence-Based Complement. Altern. Med., pp. 1-17, 2013.

[31] P. Abaci, Take Charge of Your Chronic Pain: Latest research, cutting edge tools, and alternative treatments for feeling better. USA: Globe Pequot Press., 2011.

[32] \& T. H. Chen,K., Hsu Y., Chem, W., "Well-being of institutionalized elders after yang style tai chi practice," J. Clin. Nursing, pp. 845-52, 2007.
Tearse, R. G., \& Weimer, "Tai Chi and SelfRated Quality of Sleep and Daytime Sleepiness in Older Adults: A Randomized Controlled Trial," $J$. Am. Geriatr. Soc., vol. 52, no. 6, pp. 892-900, 2004.

[34] P. Crombez, G., Vervaet, L., Lysens, R., Baeyens, F., \&Eelen, "Avoidance and confrontation of painful, back-straining movements in chronic back pain patients," Behav. Modif., vol. 22, no. 1, pp. 62-77, 1998.

[35] C. Ostelo, R. W., Deyo, R. A., Stratford, P., Waddell, G., Croft, P., Von Korff, M., ...\& Henrica, "Interpreting change scores for pain and functional status in low back pain: towards international consensus regarding minimal important change," Spine (Phila. Pa. 1976)., vol. 33, no. 1, pp. 90-94, 2008.

[36] C. J. Foster, N. E., Thomas, E., Bishop, A., Dunn, K. M., \& Main, "Pain," vol. 148, no. 3, pp. 398406, 2010. 